\title{
Kinematic cues for the categorization of pointing movements made by hemiparetic stroke patients.
}

\author{
Agnès Roby-Brami*, $\uparrow$, Lorena Borjas*, Sylvain Hanneton* \\ (*)University Paris Descartes, CNRS UMR 8119, 75006, Paris, France. \\ (†) Raymond Poincaré Hospital, 92380 Garches, France \\ E-mail: agnes.roby-brami@parisdescartes.fr,loretta503@hotmail.com, \\ sylvain.hanneton@parisdescartes.fr
}

\begin{abstract}
Our visual sensitivity to human movements is illustrated by our ability to recognize actions even with a minimal visual display. The present study investigates if professional expertise influences the precision of recognition and the visual cues used. We presented movements made by hemiparetic stroke patients and healthy subjects to expert (physicians and physiotherapists) and naive observers. Since stroke induces perturbations of hand trajectory and interjoint coordination, we presented two types of computer animation (one dot representing the hand and stick diagram of the upper body). The observers had to score the pathological character of the movements.

The observers were able to differentiate patients according to their impairment level. The experts responded faster but, surprisingly, their responses did not differ from those of naïve observers. The subjective judgment relied mainly on the quality of hand trajectory. The weak influence of postural information should be confirmed with more realistic displays.
\end{abstract}

\section{Introduction}

When observing other people we are able to understand and interpret their actions due to a particular sensitivity to human movement [1]. This corresponds to the hypothesis of a common coding mechanism for action and action recognition. One influential interpretation lies in the observation in monkeys of mirror neurons which fire when the monkey performs an action and when it watches the experimenter performing the same action [2].

The subjective rating of movement by observers has received much attention. There is evidence that observers may perceive dynamic information relating to actions from the observation of kinematics. For example, the weight of lifted objects can be recognized from simple point light displays [3]. Observers can also recognize general information about the person from the observation of their movements: e.g. gender [4] or context related expectations [5]. Along this line, our aim is to investigate the ability of observers to categorize the "pathological" level of movements thanks to minimal kinematic displays. Since the degree of familiarity with the motor repertoire observed is important [6] we compared expert (clinicians used to movement recordings) and naive observers. We focus on the movements made by hemiparetic patients which are both well characterized and variable from a patient to another. Following stroke, motor disability is due to several impairments (muscle weakness and spasticity, loss of inter-joint co-ordination and incorrect timing which contribute to alterations in the trajectories of goal-directed movements. In addition, the patients may use compensation such as trunk flexion $[7,8]$. The scores of clinical scales thus reflect both the consequences of impairments and the development of compensatory strategies.

\section{Methods}

\subsection{Participants}

20 "observer" participants (10 experts and 10 naive) took part in this experiment which lasted approximately 40 minutes. The expert observers were clinicians (MD or physiotherapists) experimented in 3D movement recording (age 25-72 mean 38.6, two females); the naive observers were neither familiar with stroke patients nor with human movement recordings (age 28 to 40, mean 28.3, four females). All the participants had normal or corrected-to-normal vision. All the experiments were performed in accordance with the ethical standards laid down in the 1991 Declaration of Helsinki.

This is an Open Access article distributed under the terms of the Creative Commons Attribution-Noncommercial License 3.0, which permits unrestricted use, distribution, and reproduction in any noncommercial medium, provided the original work is properly cited. 


\subsection{Movement recording and display.}

The "actors" for the movement recordings were 3 healthy subjects and 12 patients with hemiparesis following a stroke $(8$ with right and 6 with left hemisphere damage, age 28- 79 mean 56.25, two females). All healthy subjects (age 42-56, mean 49, two females) were right handed. Scores of routine clinical tests were used to quantify the level of impairment and functional ability: Action Research Arm Test (ARAT) and Box and Block test [9].

For the recordings, the participants were seated on a chair in front of a table, approximately level with the navel. The task consisted of making three reciprocal movements to a target placed on a removable support $17 \mathrm{~cm}$ above the table at $90 \%$ of arm length. The starting position was with the hand on the abdomen. Healthy subjects performed with their right hands, the patients with their hemiparetic arms.

Figure 1 Range of impairment in the 12 patients. Diagram giving ARAT and BBT scores.

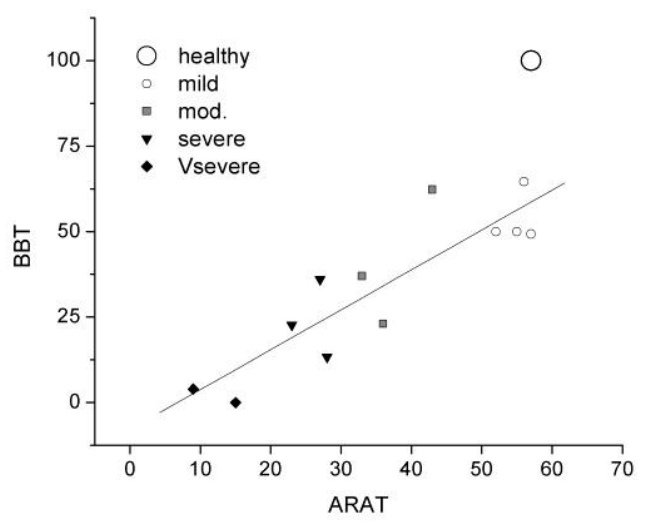

Recordings were made using an electromagnetic Polhemus system (acquisition frequency $=30 \mathrm{~Hz}$ ) with 4 sensors attached on the manubrium, the acromion process, the upper third of the arm and on the dorsum wrist. The digitization of the trunk and upperlimb landmarks for reconstruction and the computation of joint rotations (trunk flexion/extension, upper arm elevation and elbow flexion extension) were performed according to [10].

The set of movements prepared for presentation included 3 different instances of repetitive pointing movements for each "actor" (i.e. 45 movements). Two kinds of graphical animations were prepared: the movement of a unique dot representing the hand (working point display WP), and a stick diagram of the trunk and upper limb (SD). The side view was presented in both cases.

\subsection{Observation and judgement.}

The WP and SD visualization were carried out in two sessions in a counterbalanced order. The observers were asked to rank the estimated quality of movement on a visual-analog scale from 0 (highly pathological) to 100 (healthy). They gave their response using the computer mouse in a forced choice paradigm. No feedback was given. Before the start of the experiment, the participants were shown a practice sample of 15 movements in each condition. The response was recorded, as well as the time taken to respond. The whole experiment lasted about 40 minutes.

\subsection{Kinematic analysis}

The following 11 kinematic variables were measured for each of the 3 repetitive pointing movements toward the target (peak velocity, mean acceleration, number of velocity peaks, curvature index, duration, delay between go and return, hand movement distance, amount of scapula displacement, trunk flexion, arm elevation and elbow extension). A principal component analysis (PCA), with normalized varimax rotation, was performed on the 10 variables obtained for the 45 movements.

\subsection{Analysis of the subjective responses}

The responses correspond to the visual rating of the impairment level by the observers were first analyzed according to the clinical level of impairment. Repeated measures ANOVA were performed with observer group (expert/non-expert) and display type (WP/SD) as factors and ANCOVA with the scores of ARAT and BBT as cofactors. Simple and Multiple Stepwise Regression analyses were performed to calculate the percentage of the variation of the responses due to the kinematics with Factors given by the PCA as independent variables.

\section{Results}

\subsection{Kinematics of patient's movements}

The patients were selected in order to present a wide range of motor capacity (Figure 1). Their movements were slower than those of healthy subjects, with increased curvature and decreased smoothness. They used less elbow extension for reaching. The patients with a severe impairment were separated in two groups depending on the presence (severe 1) or absence (severe 2) of compensatory trunk flexion. The kinematic variables were very roughly scaled to the level of impairment with a great variability. 
4. Subjective response on the impairment. Repeated measures ANOVA demonstrated that the observers were able to scale the level of impairment (Figure 2 upper part, F288,4=106.3, $\mathrm{p}<0.0001$ ).

Surprisingly, the responses made by the expert and non-expert observers were similar (F288,4=0.12, ns). The type of display did not influence the response $(\mathrm{F} 288,4=1.6, \mathrm{~ns})$ and there were no interactions. The observers were able to discriminate healthy subjects from patients (post hoc Tukey-Kramer) and patients with a mild impairment from the others. However, they did not discriminate patients with a moderate or severe impairment. ANOVA showed that the experts respond faster $(\sim 3 \mathrm{~s})$ than the non-experts $(\sim 3.5$ to $4 \mathrm{~s})$ (Figure 2 lower part, $F 288,4=17.2, \mathrm{p}<0.0001)$. There were no significant difference with the level of impairment $(\mathrm{F} 288,1=0.8, \mathrm{~ns})$ or the type of display $(\mathrm{F} 288,1=2.3$, ns) nor interactions between factors.

Figure 2 Response and response time as a function of the impairment level.

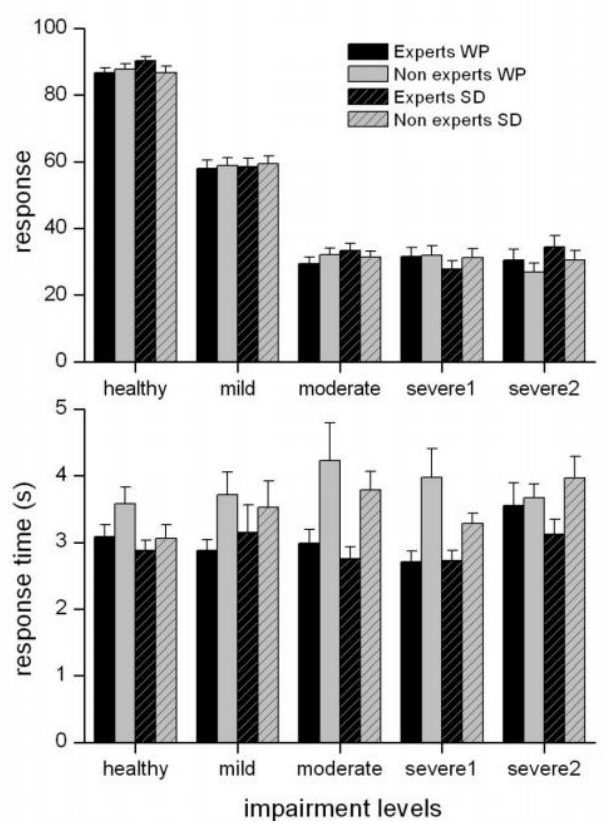

The ability of observers to discriminate the level of impairment was confirmed by a regression analysis of the response as a function of ARAT and BBT after exclusion of healthy subjects ( $\mathrm{p}<0.0001$ in all cases). ARAT score explained $24 \%$ and $20 \%$ of the variance of the experts' responses and 30 and $27 \%$ of the nonexperts' responses (WP and SD display respectively). BBT score, explained $19.7 \%$ and $15.3 \%$ of the experts' responses and 26.9 and $23.2 \%$ of the nonexperts' responses (WP and SD display respectively).

\subsection{Subjective response and kinematics.}

PCA was performed to summarize the kinematic variables. It yielded 3 factors which explained a total of $84.2 \%$ of the variance (Table 1). The first factor was correlated to the variables related to the quality of the hand trajectory. The second factor was likely related to subject size and the third to the presence of compensatory trunk flexion. Velocity and acceleration were moderately correlated to all three factors.

Table 1 PCA of the kinematic variables

$\begin{array}{lccc} & \text { Factor 1 } & \text { Factor 2 } & \text { Factor 3 } \\ \text { eigenvalue } & 6.48 & 1.76 & 1.02 \\ \text { \% variance explained } & 58.94 & 15.96 & 9.29 \\ \text { Cumulated variance } & 58.94 & 74.90 & 84.20 \\ & & & \\ & \text { Quality of } & \text { Subject's } & \text { Trunk } \\ & \text { trajectory } & \text { size } & \text { compensation } \\ \text { Peak vel } & -0.666 & 0.448 & 0.378 \\ \text { mean acc } & -0.625 & 0.433 & 0.429 \\ \text { number peaks } & \mathbf{0 . 9 5 0} & -0.062 & -0.140 \\ \text { curv. index } & \mathbf{0 . 9 3 9} & -0.100 & -0.125 \\ \text { duration } & \mathbf{0 . 8 8 3} & -0.181 & -0.359 \\ \text { delay } & \mathbf{0 . 8 1 6} & -0.188 & -0.111 \\ \text { mov. distance } & -0.350 & \mathbf{0 . 8 7 2} & 0.078 \\ \text { scap. displacement } & 0.197 & -0.256 & -0.895 \\ \text { trunk flexion } & 0.250 & -0.188 & -0.885 \\ \text { arm elevation } & 0.136 & \mathbf{0 . 8 1 8} & 0.282 \\ \text { elbow extension } & -0.432 & \mathbf{0 . 7 3 1} & 0.249\end{array}$

Figure 3 Multiple regression analysis of the response as a function of kinematic variables.

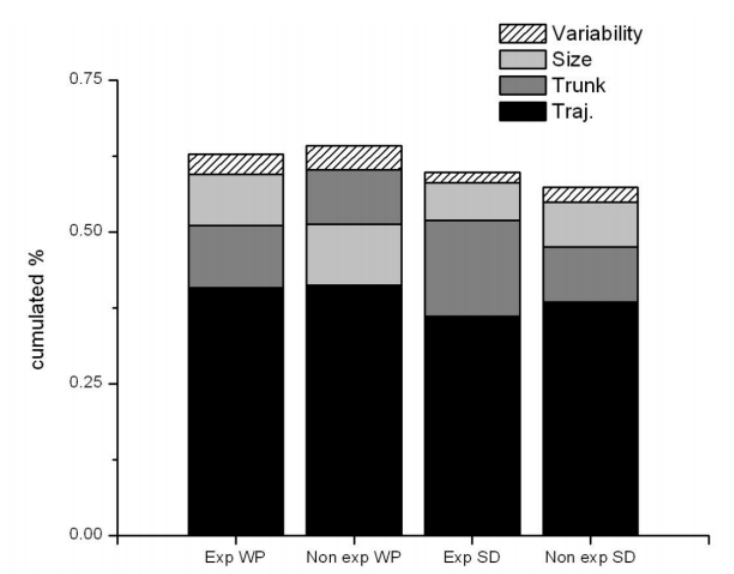

Multiple stepwise analysis was performed on the responses with as independent variables: the three PCA factors as independent variables, more a measure of movement variability (Figure 3 ). PC1, representing the quality of the trajectory, explained the most variance in all the conditions. Simple regression analyses were performed separately for each observer in order to obtain statistical differences. PC1 explained $39-45 \%$ of the response whatever the type of display $(\mathrm{F} 18,1=0.9$, 
$\mathrm{ns})$ or the group $(\mathrm{F} 18,1=0.3$, ns). The variability of movements across repetition explained $27-30 \%$ of the responses according to the condition, with no significant differences with the type of display $(\mathrm{F} 18,1=$ $0.5, \mathrm{~ns})$ or between groups $(\mathrm{F} 18,1=0.2, \mathrm{~ns})$. The factor representing size explained $\sim 9 \%$ of the response in PT condition, and $\sim 7 \%$ in SD condition, but the difference was not significant $(\mathrm{F} 18,1=3.2$, ns $)$. The factor representing trunk flexion represented $\sim 9 \%$ of the response with a notable exception in the case of expert observing movements with SD display $(17 \pm 9 \%$ of the response). The effect of the display was significant $(\mathrm{F} 18,1=5.35, \mathrm{p}=0.03)$, but not the group effect $(\mathrm{F} 18,1=$ $3.52, \mathrm{~ns})$ or the interaction $(\mathrm{F} 18,1=3.50, \mathrm{~ns})$.

\section{Discussion}

In brief, this study demonstrates i) that observers are able to recognize impaired movements from observation and ii) that the most pertinent cues are linked to the quality of hand trajectory. The other dimensions of the movements (size, posture and regularity) play a much smaller role.

The observers, whatever expert or not, easily discriminated the healthy subjects from the patients, with a mild impairment. This fact may contribute to the discrimination and social handicap of patients with a good functional level. In contrast, observers were unable to differentiate between patients with moderate or severe impairment. This may be due to the fact that clinical scores involve grasping while we presented pointing movements.

In contrast to what we anticipated, the compensatory trunk movements were little used for categorization (e.g; the severe 1 and severe 2 groups of patients were scaled similarly). However, the SD display was quite crude. The study should be performed with more realistic presentations (avatars) before concluding.

The experts were faster to respond, perhaps because they felt more confident but, surprisingly their responses generally did not differ from those of non experts. The only tendency suggested that the experts were more sensitive than non-experts to the postural cues presented (i.e. cues related to compensatory trunk flexion visible on SD display).

The fact that the experts were not "better" for recognition is surprising. A study in dancers showed that personal habits of the motor repertoire were particularly important for brain activation during action observation [6]. A similar study is in progress with hemiparetic patients as observers to test this point.

\section{Acknowledgements}

The authors thank Thomas Hollinger for technical assistance, Johanna Robertson for discussing the clinical evaluation and all the expert and non-expert participants. This research was supported by a grant from the European Communities: NEST project Handto-Mouth contract $n^{\circ}$ 029065. Agnes Roby-Brami is supported by INSERM.

\section{References}

[1] P. Viviani, and N. Stucchi. Biological movements look constant: Evidence of motor-perceptual interactions. Journal of Experimental Psychology: Human Perception and Performance, 18, 603-623, 1992

[2] G. Rizzolatti, and L. Craighero. The mirror-neuron system. Annual Review of Neuroscience, 27, 169-192, 2004.

[3] J. Shim, L.G. Carlton, and J. Kim. Estimation of lifted weight and produced effort through perception of pointlight display. Perception, 33, 277-291, 2004.

[4] F.E. Pollick, J. W. Kay, K. Heim, and R Stringer. Gender recognition from point-light walkers. Journal of Experimental Psychology: Human Perception and Performance, 31, 1247-1265, 2005.

[5] A. Jacobs, J. Pinto, and M. Shiffrar. Experience, context, and the visual perception of human movement. Journal of Experimental Psychology: Human Perception and Performance, 30, 822-835, 2004

[6] B. Calvo-Merino, D.E. Glaser, J. Grèzes, R.E. Passingham, and P. Haggard. Action observation and acquired motor skills: an FMRI study with expert dancers. Cerebral Cortex, 15: 1243-1249, 2005.

[7] M.C. Cirstea, and M.F. Levin Compensatory strategies for reaching in stroke. Brain, 123: 940-953, 2000

[8] A. Roby-Brami, A. Feydy, M. Combeaud, E.V. Biryukova, B. Bussel, and M.F. Levin. Motor compensation and recovery for reaching in stroke patients. Acta Neurologica Scandinavica, 107: 369-381, 2003

[9] T. Platz, C. Pinkowski, F. van Wijck, I.H. Kim, P. di Bella, and G. Johnson. Reliability and validity of arm function assessment with standardized guidelines for the Fugl-Meyer Test, Action Research Arm Test and Box and Block Test: a multicentre study. Clinical Rehabilitation, 19: 404-411, 2005

[10] S. Hanneton, S. Dedobbeler, T. Hoellinger, and A. Roby-Brami, Direct kinematic modeling of the upper limb during trunk-assisted reaching. Journal of applied biomechanics. 27:272-277, 2011. 